\title{
La reestructuración en la industria: los casos de Chile, México y Venezuela
}

\section{Carla Macario}

División de Desarrollo

Productivo y Empresarial, CEPAL
Las economías de América Latina experimentaron importantes transformaciones en años recientes. Aunque ha habido muchos estudios sobre los cambios macroeconómicos que han tenido lugar en la región, son relativamente escasos los referidos a los cambios microeconómicos. Este artículo aporta antecedentes acerca de cómo responden las empresas a circunstancias nuevas. La investigación en empresas manufactureras latinoamericanas demuestra que el nuevo entorno económico ha modificado el comportamiento de las empresas. Las empresas innovadoras han adoptado comportamientos flexibles y están mejorando su capacidad de producción y comercialización, y han introducido cambios importantes en materia de integración vertical, abastecimiento de insumos, innovación tecnológica, sistemas de incentivos salariales y técnicas de gestión, capacitación, subcontratación, distribución y venta al detalle. Al menos para los fabricantes más innovadores de bienes de consumo, la esencia del negocio no es ya la producción, sino una combinación de fabricación y distribución de este tipo de bienes, muchas veces agregando a los propios otros productos nacionales e importados. Las empresas tienen así más probabilidades de aumentar sus ganancias junto con defender su participación de mercado. Por último, la investigación mostró también que cuando la incertidumbre rodea a la política económica, la inversión de las empresas disminuye considerablemente. Esa incertidumbre hace que muchas de ellas no cambien o que lo hagan con lentitud, y tiene un doble efecto negativo en las decisiones de modernización de los empresarios: éstos tienen dudas tanto de lo que deben hacer, como de la sustentabilidad de la política económica. Por lo tanto, es importante que la política se traduzca en programas para estimular el mejoramiento de las empresas. Al mismo tiempo, debe destacarse que la función más importante de la política es la de crear un clima económico estable en el que las empresas puedan planificar la inversión de largo plazo. 


\section{I}

\section{Introducción}

Los países de América Latina han sufrido importantes transformaciones en los últimos años. Ha habido cambios radicales en sus políticas macroeconómicas y de comercio y en el entorno económico general, así como en materias relacionadas con la intervención del Estado y el marco regulatorio. A todo ello se suma una creciente globalización de los mercados mundiales. Como resultado, se han registrado cambios importantes en el plano macroeconómico y en la forma de inserción de las economías de la región en la economía mundial, así como en el comportamiento de las empresas.

Aunque se ha escrito mucho sobre los cambios macroeconómicos ocurridos en América Latina, son relativamente escasos los estudios que tratan de los cambios microeconómicos. Uno de los primeros de estos estudios analizaba cómo las empresas chilenas se adaptaron a esos cambios durante el decenio de 1970 y principios del de 1980 (Corbo y Sánchez, 1984). Más recientemente, y habiéndose llegado a cierto consenso en la mayoría de los países respecto de las políticas macroeconómicas que deberían adoptarse para lograr la estabilidad en el largo plazo, ha habido un interés creciente en investigar las modificaciones en el comportamiento de las empresas manufactureras y la forma en que se están adaptando al nuevo entorno económico (Baumann, 1994; Bielschowsky, 1994; Castillo, Dini y Maggi, 1994 y Katz y Burachik, 1997).

Este artículo presenta los resultados de una investigación sobre las estrategias de las empresas manufactureras y sus cambios como consecuencia de la liberalización, la globalización y la transformación del medio económico en que operan. Las principales preguntas que se planteó esta investigación fueron: ¿Están cambiando sus estrategias las empresas del sector tradicional manufacturero de bienes de consumo, o siguen funcionando en general como hace 20 años? ¿Qué tan determinante es la influencia de los acontecimientos macroeconómicos en los microeconómicos, y cuál

$\square$ La autora agradece a Ricardo Bielschowsky, Jorge Katz y Joseph Ramos los valiosos comentarios que llevaron a la preparación de este artículo. La investigación básica se efectuó en virtud del Proyecto CEPAL/PNUD sobre Innovación y Competitividad; las entrevistas fueron realizadas por Wilson Peres y Martine Guerguil, funcionarios de la CEPAL, y por la autora. es el margen de las empresas para el comportamiento individual? Si el comportamiento de las empresas tradicionales fabricantes de bienes de consumo está cambiando, ¿cuáles son las áreas más importantes en que dicho cambio se está verificando? ¿Cuáles son las estrategias de las empresas del sector que van a la vanguardia del proceso de modernización en la región?

La investigación se llevó a cabo en empresas tradicionales medianas y grandes que fabrican bienes de consumo, en Chile, México y Venezuela. Desde el principio, se decidió concentrarse en tales empresas por considerarse que estaban entre las más representativas de las empresas manufactureras medianas y grandes que podían encontrarse en los tres países. Los sectores incluidos abarcaban una amplia gama de industrias, pero las empresas de vestuario parecieron las más idóneas para comparar comportamientos de industrias similares entre los países, porque pertenecen a una actividad establecida hace varias décadas, no sólo en los países incluidos en el estudio, sino que también en los demás países latinoamericanos. Además, era interesante estudiar esta industria porque estaba fuertemente expuesta a la competencia de las importaciones y por el impacto de la globalización en la industria mundial del vestuario.

El estudio incluyó más de 40 empresas del vestuario, aunque sólo se logró información comparable para $38 ;{ }^{1}$ de modo que las conclusiones presentadas en este artículo están inferidas fundamentalmente de empresas de este rubro. ${ }^{2}$ Las entrevistas a ejecutivos de las empresas se complementaron con cuestionarios abiertos, visitas a las plantas, y reuniones con representantes de asociaciones industriales, expertos industriales y funcionarios de gobierno. Se escogieron las empresas procurando diversidad, pero la muestra no alcanza a ser representativa. La mayor parte de las entrevistas se realizaron en 1993, aunque algunas se hicieron en 1994 y 1995. En algunos casos, las entrevistas iniciales se complementaron con visitas de se-

\footnotetext{
1 Véase Macario (1998a).

2 No obstante, parece ser válida para otras empresas tradicionales productoras de bienes de consumo, como las del cuero y el calzado (véase Macario, 1998b).
} 
guimiento para obtener una mejor perspectiva de los cambios en la empresa a través del tiempo.

Este artículo describe en la sección siguiente las principales características del comportamiento de las empresas tradicionales productoras de bienes de consumo en el esquema de sustitución de importaciones, basándose parcialmente en las entrevistas con ejecutivos de las empresas, a los que se les preguntó sobre la historia de sus empresas (sección II). Luego presenta los principales resultados de la investigación en relación con el comportamiento de una empresa tradicional manufacturera de bienes de consumo en proceso de modernización (sección III), y finalmente entrega las conclusiones más relevantes (sección IV).

Por último, una advertencia: aunque hay muchas definiciones e indicadores para medir la competitividad, en este artículo el concepto "competitivo" se usa como sinónimo de "modernizador": cuando una empresa se define como competitiva, debe entenderse como una empresa innovadora, que se esfuerza por cambiar y sobrevivir, que trata de encontrar las formas más adecuadas para adaptarse al nuevo entorno y que, como resultado, gana participación de mercado.

\section{II \\ El comportamiento de las empresas en el esquema de sustitución de importaciones}

¿Cuáles son las estrategias principales de una empresa que fabrica bienes de consumo bajo el esquema de sustitución de importaciones? En esta sección se intenta contestar esta pregunta, describiendo las características principales de la evolución de una empresa representativa.

La típica empresa entrevistada para este estudio es de tipo familiar, fundada como un pequeño taller en sectores como los del vestuario o del calzado o en una pequeña planta de moldeado de metales. Este pequeño taller se expandió lentamente, comprando nuevo equipo, trasladándose a nuevas dependencias y llegando a convertirse en empresa manufacturera.

La transición de taller a planta manufacturera fue a menudo bastante difícil y riesgosa, frente a la competencia de muchos otros talleres similares. Por lo general no existía apoyo financiero para las empresas pequeñas, de modo que uno de los factores críticos de esta transición era la capacidad de acumular recursos financieros suficientes para expandirse. De ahí que muy pocos talleres lograran transformarse en empresas manufactureras, a menos que los empresarios lograran obtener fondos suficientes, muchas veces gracias al apoyo familiar, al cabildeo en oficinas gubernamentales o a la pura suerte.

Por lo general la producción se organizaba de manera similar que en el taller, evolucionando lentamente a un patrón basado en sus líneas generales en la organización del trabajo en plantas de países industrializados.
El diseño de la planta con frecuencia lo hacía el propio empresario. La empresa fabricaba una amplia gama de productos de diseño y estándares de calidad poco sofisticados. Esto bastaba para satisfacer a una gran masa de consumidores que sólo recientemente comenzaban a tener acceso a bienes manufacturados y que, por ello, no eran muy exigentes.

La administración recaía por lo general en los miembros de la familia y no se ajustaba a criterios profesionales. Los trabajadores productivos recibían salarios fijos o, en algunos casos, salario por pieza, sin requisitos de calidad estrictos. Las empresas no daban capacitación.

La mayor parte de las operaciones de producción se hacían internamente en la empresa. La subcontratación era muy poco frecuente y sólo se utilizaba para operaciones específicas que fueran muy complejas de llevar a cabo en la propia planta o para satisfacer aumentos inesperados de la demanda.

Por lo general, los insumos se manufacturaban en el país, dadas las políticas prevalecientes de sustitución de importaciones y los costos y largos plazos implícitos en la importación. Las empresas más grandes tendían a integrarse verticalmente, fabricando insumos para su propio uso, así como para la venta a otras empresas locales. Había un poderoso incentivo para que las empresas se integraran tanto como fuera posible, ya que las que vendían bienes intermedios eran competidoras en el mercado de productos finales.

La mayoría de los bienes producidos se vendían en el mercado interno. Por lo general, era difícil para 
los productores de bienes de consumo exportar en forma regular, debido a una combinación de factores como el sesgo antiexportador derivado de los altos aranceles y de las barreras no arancelarias; el bajo nivel relativo del tipo de cambio prevaleciente en la mayoría de los países durante casi todo el período de sustitución de importaciones, y los costos excesivos del transporte dentro de la región.

Las barreras arancelarias y no arancelarias de otros países latinoamericanos que podrían haber sido los mercados más atractivos, contribuían también a restarle incentivos a la exportación. El proteccionismo y los costos de transporte hacían difícil exportar a los países industrializados.

En su mayoría, las empresas exportaban en forma ocasional a países vecinos, especialmente a aquellos con un menor grado de desarrollo industrial. Las exportaciones eran contracíclicas, aumentando cuando caía la demanda interna o cuando se depreciaba el tipo de cambio. Los productos que se exportaban eran los mismos que las empresas vendían en el mercado local. El régimen de incentivos alentaba a la mayoría de las empresas de bienes de consumo a especializarse en la manufactura para el mercado interno, protegidas de las presiones de la competencia externa.

Cuando habían logrado superar las restricciones financieras iniciales y habían alcanzado un cierto umbral de producción, las empresas lograban acceder con relativa facilidad al apoyo financiero del gobierno, que otorgaba créditos a bajas tasas de interés, a menudo subvencionadas. Esto les permitía seguir expandiéndose. Una vez que las empresas llegaban a esta etapa, las presiones para aumentar la productividad no eran tan fuertes como en la etapa anterior.

Además, cuando la empresa había establecido una planta de producción de una escala apropiada para la dimensión del mercado interno, se reducían considerablemente las presiones competitivas de empresas locales similares. El tamaño reducido del mercado interno permitía a menudo que fuera controlado por un pequeño número de empresas cuyo comportamiento se caracterizaba por la colaboración, dividiéndose entre ellas las participaciones de mercado, que permanecían bastante estables en el tiempo.

Así, una vez que la empresa alcanzaba una escala de producción mínima y una determinada participación en el mercado interno, el fabricante típico de bienes de consumo llegaba a una suerte de meseta. A la empresa le era perfectamente posible seguir funcionando de esa manera; con un estilo familiar de administración no había presiones importantes para que la empresa siguiera evolucionando, y su entorno llegaba a ser bastante estable y seguro.

Al traspasar un determinado umbral, la falta de presiones competitivas repercutía en la forma en que se organizaba la producción. De ahí que, mientras la producción se organizara siguiendo a grandes rasgos los patrones de los países industrializados, las empresas no necesitaban continuar introduciendo cambios para mejorar la productividad y la calidad.

El modelo de desarrollo basado en la sustitución de importaciones permitió a la región crear un sector manufacturero. Sin él, la mayoría de los países hoy tendrían una cantidad mucho menor de empresas industriales. Este modelo proporcionó las condiciones de aprendizaje y evolución que hicieron posible transformar el taller en empresa manufacturera. Contribuyó también a moldear a varias generaciones de inmigrantes rurales, transformándolos en trabajadores de la producción industrial. A mayor abundamiento, una parte importante de los bienes manufacturados que hoy exporta la región proviene de empresas que existen gracias a las políticas de sustitución de importaciones. Sin embargo, hacia el decenio de 1960 este modelo llegó a ser un impedimento para el desarrollo de los países latinoamericanos (CEPAL, 1994).

Entre mediados de los años ochenta y fines de los noventa, la mayoría de los países de la región transformaron significativamente las políticas macroeconómicas y de comercio, así como la mayor parte del marco regulatorio. Estos cambios tuvieron un impacto considerable en las empresas, al modificar el esquema al que estaban habituadas y someterlas a las fuertes presiones competitivas de las importaciones. 


\section{III}

\section{El comportamiento de las empresas}

\section{en los años noventa: Principales resultados de la investigación}

\section{Influencia de las políticas macroeconómicas y comerciales}

¿Cómo influyen las políticas macroeconómicas y comerciales en el comportamiento de las empresas? Para evaluar esta influencia es útil examinar las diferencias en el comportamiento de empresas de Chile, México y Venezuela, y su evolución en cada país.

La mayoría de las empresas chilenas mantuvo sus niveles de producción o los aumentó durante el año que precedió a las entrevistas. Esto fue fruto de la estabilidad macroeconómica general y el crecimiento sostenido de Chile. También se debió al hecho de que las empresas que habían logrado sobrevivir al choque de la reestructuración industrial de fines de los años setenta y comienzos de los ochenta eran relativamente sólidas, aun bajo condiciones de fuerte competencia de las importaciones.

Las empresas mexicanas tuvieron dos tipos de comportamiento. La mayoría había visto caer su producción hasta en 30\% como consecuencia de la baja tasa de crecimiento de la economía mexicana hasta 1993 y la creciente penetración de las importaciones. Aun así, varias empresas que habían invertido vigorosamente en modernización, o que exportaban, habían aumentado su producción.

En contraste, la producción de la mayoría de las empresas venezolanas había tenido una fuerte caída debido a la importante disminución de la demanda por la incertidumbre política de la última parte de 1992 y la fuerte competencia de las importaciones.

Los cambios macroeconómicos parecen haber llegado a ser más importantes para las empresas manufactureras después de la liberalización del comercio. Por ejemplo, una apreciación del tipo de cambio se traduce en un aumento considerable de la competencia de las importaciones en un período muy corto de tiempo.

Otra comprobación del impacto de las tendencias macroeconómicas en el comportamiento de las empresas es la evolución de las exportaciones de las empre- sas chilenas. Mientras en 1990 varias de las empresas investigadas en este país pertenecían a una categoría que podríamos denominar de "exportación moderada" y exportaban por lo regular entre el $5 \%$ y el $10 \%$ de su producción, en 1992 ya no quedaban empresas de este tipo en Chile: o habían desertado del mercado de exportación o habían aumentado significativamente su esfuerzo exportador y el porcentaje de su producción que exportaban. El crecimiento de la demanda interna, combinado con la apreciación del tipo de cambio, polarizó el comportamiento exportador de las empresas, impulsando a la mayoría a concentrarse exclusivamente en el mercado interno; las que quisieron seguir exportando debieron especializarse crecientemente en el mercado de exportación. Esto se ilustra por el hecho de que mientras entre las empresas chilenas entrevistadas en ciertos sectores el número de exportadoras decreció al apreciarse el tipo de cambio, las exportaciones medias de las empresas que exportaban habitualmente aumentó del $15.6 \%$ de su producción en 1990 al 44\% en 1992.

El hecho de que en la producción de las empresas influyeran acontecimientos macroeconómicos muestra la gravitación del entorno macroeconómico en el comportamiento de las empresas.

De la misma forma, hay una vinculación clara entre el comportamiento exportador y el grado de apertura de la economía: ninguna de las empresas entrevistadas en Venezuela estaba exportando en el momento de las entrevistas (marzo de 1993); sí lo hacían empresas de Chile y muchas más de México. Estos dos países habían liberalizado su comercio antes que Venezuela y sabían que no habría un retroceso en la política. Venezuela, en cambio, había liberalizado su comercio sólo a fines del decenio de 1980 pero, en el momento de las entrevistas, se suscitaban serias dudas sobre la continuación de esta política. Los acontecimientos que tuvieron lugar en Venezuela con posterioridad confirmaron la validez de esta apreciación.

La fuerte influencia de las políticas macroeconómicas y de comercio en el comportamiento de las 
empresas significa que las consecuencias de esas políticas deben ser evaluadas con mucho cuidado. Asimismo, es preciso tener presentes los efectos en el comportamiento de las empresas de la incertidumbre sobre la política económica, como sucedió en Venezuela a partir de 1993.

\section{Margen para el comportamiento microeco- nómico}

No obstante, pese a la importante influencia del entorno económico sobre las empresas, la investigación muestra que hay margen para la estrategia empresarial: no todas las empresas chilenas estudiadas eran modernizantes ni todas las venezolanas les iban a la zaga.

Algunas empresas lograron competir a pesar de un ambiente adverso y de pertenecer a un subsector con fuerte competencia de las importaciones. Como ejemplo destacan dos empresas venezolanas que estuvieron dispuestas a seguir invirtiendo, capacitando a su personal y buscando nuevas estrategias de sobrevivencia, mientras la mayoría de las empresas de ese país veían caer su producción y su participación de mercado debido a la recesión y a la competencia de las importaciones. Estas empresas modernizantes habían apostado a sobrevivir y estaban emprendiendo una estrategia activa de modernización para llegar a ser competitivas.

Otro ejemplo es el de varias industrias mexicanas que, como las de vestuario y del calzado, enfrentaban una competencia particularmente fuerte de las importaciones. Mientras muchas empresas de estas industrias cerraron, varias sobrevivían bastante bien, gracias a sus esfuerzos por adaptarse al nuevo entorno.

Deberían investigarse más a fondo las estrategias de las distintas empresas y su capacidad de aprender y adaptarse a nuevos entornos. Hay mucho que aprender de ellas, y saber más de sus estrategias sería de utilidad para el diseño de políticas. También debería intentarse proveer a la teoría económica de un marco teórico más sólido para analizar el alcance del comportamiento microeconómico (Nelson, 1991).

\section{Cambios en el comportamiento de las empre- sas}

La descripción del comportamiento general de una empresa manufacturera en un régimen de sustitución de importaciones calza muy bien con el de algunas empresas de hoy. No obstante, la investigación llevó a concluir que el comportamiento de muchas empresas está sufriendo cambios significativos.
Las entrevistas con ejecutivos de las empresas y representantes del sector privado mostró que el ambiente empresarial es muy dinámico y que se están registrando cambios. Es posible que estén ocurriendo muchas más transformaciones que las que se pueden percibir a nivel global, en parte porque algunas son todavía incipientes y muchas han tomado direcciones opuestas.

Para sobrevivir, las empresas manufactureras se han visto obligadas a adaptarse a su nuevo entorno. Esto ha requerido significativas inversiones, que pueden ser de muy alto costo por la disponibilidad limitada de financiamiento de largo plazo y por las altas tasas de interés que han prevalecido en la región, especialmente bajo políticas de estabilización.

De acuerdo con la información recolectada en esta investigación, ¿cuáles son los cambios actuales en las empresas manufactureras de bienes de consumo en Chile, México y Venezuela? Para responder, primero describiremos el comportamiento de la mayoría de las empresas modernizantes para luego centrar la atención en algunas áreas específicas donde ha habido cambios mayores.

Las empresas modernizantes se están especializando: reducen el número de líneas de producción, y a la vez aumentan el número de productos dentro de las líneas que mantienen. Están achicando el tamaño de los lotes y el tiempo que les toma producirlos. Están también disminuyendo inventarios, especialmente los de bienes finales.

Para lograr sus propósitos, están modificando su diseño de planta, siguiendo el consejo de consultores externos que ellas mismas contratan o que contactan a través de compañías extranjeras, tales como clientes o empresas licenciatarias. Los cambios en el diseño de planta ya no se conciben como duraderos; más bien se aplican con flexibilidad y hay disposición a adaptarlos regularmente según los requerimientos de la demanda.

Los cambios en el diseño de planta tienen por objetivo facilitar el flujo de la producción y mejorar el control de calidad. La inclusión de controles automáticos de flujo permite a la empresa tener información precisa sobre la productividad de un trabajador individual y detectar con rapidez los cuellos de botella.

Las plantas que se están modernizando más exitosamente son las que han sido capaces de aplicar estándares de producción y metas de eficiencia de acuerdo con los procedimientos fordistas. Esto les ha permitido aumentar enormemente la productividad. Las rutinas de producción se han modificado también para 
incluir más puntos de control de calidad y, en la mayoría de las plantas avanzadas, para aumentar la responsabilidad del trabajador individual en lo que toca a estándares de calidad. En lo que respecta a determinar si la especialización flexible y la mayor autonomía de los trabajadores están reemplazando a los procedimientos fordistas, la investigación confirma lo reseñado en otro estudio de casos en la industria brasileña del calzado que sugiere que más importante que los límites son las conexiones entre la especialización flexible y el fordismo (Schmitz, 1995).

La calidad se ha elevado sustancialmente gracias a un importante mejoramiento de la capacidad de diseño a medida que se contrata personal profesional y se instalan equipos automatizados.

El deseo de mejorar la calidad y de alcanzar metas de eficiencia ha llevado a las empresas a modificar sus sistemas salariales. Los cambios puestos en vigor para este propósito y que se describen a continuación muestran que ellas buscan sistemas de remuneración que se ajusten a sus necesidades de elevar los estándares de productividad y calidad.

Las modificaciones en la organización de la producción y en los sistemas de remuneraciones se acompañan con cambios en los métodos de administración, a medida que las empresas pasan de una administración de estilo familiar, a una basada en criterios profesionales. Estos cambios responden a un medio crecientemente competitivo, pero a menudo coinciden con una transición generacional en la propiedad.

Las áreas en que se están verificando los cambios más importantes son las que se señalan a continuación.

\section{a) Integración vertical}

Ilustrativo de los cambios en las políticas macroeconómicas y de comercio que se han traducido en cambios a nivel microeconómico, es el grado de integración vertical dentro de las empresas. Bajo las políticas de sustitución de importaciones era ventajoso un alto grado de integración vertical porque las empresas que vendían bienes intermedios tenían un apreciable poder en el mercado, especialmente cuando vendían a empresas rivales en el mercado de bienes finales. Sin embargo, éste ya no es el caso, por el aumento de las opciones de abastecimiento de insumos desde fuera del país, fruto de la liberalización del comercio.

En efecto, las entrevistas en plantas integradas verticalmente mostraron que, en muchas industrias, un alto nivel de integración vertical, lejos de ser un activo, representa hoy una desventaja para las empresas, en la medida en que restringe su flexibilidad de abas- tecimiento. En muchas ocasiones, los gerentes a cargo de la producción de bienes finales se quejaron de tener que usar insumos de la misma empresa, obligación que restringía las posibilidades de comprar una variedad de insumos de bajo costo en un período de tiempo razonable. En la mayoría de los casos, no sólo era más barato comprar insumos externos, sino que las demoras de abastecimiento se reducían. Cada una de las empresas integradas verticalmente afirmaba que estaba utilizando proporciones cada vez menores de insumos producidos en la misma empresa.

Las plantas que fabricaban bienes intermedios no competitivos han sido particular y severamente afectadas, ya que no sólo ha disminuido la producción de insumos para las plantas de la misma empresa, sino que la demanda de sus bienes por parte de otras empresas del país se ha desplomado. Frecuentemente, a menos que produzcan un insumo muy competitivo, las plantas integradas verticalmente son un obstáculo a la flexibilidad que necesitan las empresas para funcionar y ser capaces de responder a cambios rápidos de la demanda.

Algunas empresas han empezado a enfrentar este problema estableciendo empresas diferentes dentro de un conglomerado y disminuyendo la obligación de comprar insumos en las plantas relacionadas. En muchos casos, la evolución hacia la desintegración vertical ha ido más allá, con empresas separadas y de propiedad distinta. Esto permite especializarse en segmentos distintos de la secuencia productiva, obteniendo economías de escala.

\section{b) Utilización de insumos importados}

Junto con reducirse la integración vertical ha habido un importante cambio en el abastecimiento de insumos, dado el uso creciente de insumos importados en empresas de los tres países. Esto era algo que se podía esperar luego de los procesos de liberalización del comercio combinados con la apreciación cambiaria. En los tres países, las empresas consideradas aumentaron el uso de insumos importados en los tres años anteriores.

Las empresas chilenas usaban porcentajes más altos de insumos importados que sus contrapartes mexicanas y venezolanas, lo que se debe a que la liberalización del comercio empezó en Chile antes que en los otros dos países. También es consecuencia del pequeño tamaño de la economía, que limita la variedad de insumos fabricados internamente.

Para las empresas que no estaban integradas verticalmente, la liberalización del comercio fue una opor- 
tunidad para comprar una mayor variedad de insumos, frecuentemente de mejor calidad y de menor costo. El uso de insumos importados ha tenido gran importancia en la estrategia de las empresas para ser más competitivas.

\section{c) Tecnología}

Se hizo una estimación aproximada del nivel tecnológico de las empresas, comparando el equipamiento en las entrevistas con los ejecutivos y en las visitas a las plantas.

La primera conclusión es que al comparar el nivel tecnológico de las empresas de una misma industria entre países las diferencias, sorprendentemente, son mucho menores que lo que cabría esperar, dados los diferentes tamaños de las economías. Aunque es cierto que algunas de las empresas de tecnología más avanzada eran mexicanas, la distancia media entre industrias similares de los tres países no parecía ser muy grande.

Por el contrario, se observó una alta dispersión de niveles tecnológicos en empresas de la misma industria en un mismo país. Así se vio en Chile, México y Venezuela. Esta variación muestra la heterogeneidad de la industria latinoamericana, en la cual diferentes niveles tecnológicos parecen ser capaces de coexistir lado a lado, al menos en los sectores tradicionales de fabricación de bienes de consumo. La dispersión observada era más grande en México que en los otros dos países.

Curiosamente, no hay una correlación sistemática entre el tamaño y el nivel tecnológico de las empresas grandes y medianas. Varias de las más grandes empresas entrevistadas tenían niveles tecnológicos bastante bajos, mientras que algunas de tamaño medio tenían mejor equipamiento y mejores procedimientos administrativos. Esto puede deberse a que en algunas de las empresas grandes, que son más antiguas y acostumbran funcionar en un entorno de sustitución de importaciones, se están planteando otros problemas, como el aumento de la flexibilidad y la subcontratación, antes de introducir innovaciones tecnológicas.

De la misma forma, no hay una correlación sistemática entre la inversión en equipamiento y la competitividad de la empresa. Varias firmas tenían equipos relativamente sofisticados, sin ser muy competitivas. Por ejemplo, algunas empresas, particularmente en Venezuela, tenían equipos relativamente avanzados que a menudo no utilizaban.

Sin embargo, aunque un nivel tecnológico relativamente alto no implica que una empresa sea compe- titiva, lo opuesto parece ser verdad. Más que el nivel tecnológico alcanzado, lo que parecía caracterizar a las empresas modernizantes era la búsqueda de formas de mejorar ese nivel adquiriendo el equipamiento que les permitiera resolver problemas específicos.

En otras palabras, más que el gasto en bienes de capital para la planta completa, lo que caracterizaba a las empresas modernizantes era su afán de optimizar la incorporación de tecnología en la planta, y hacerlo en forma sistemática y periódica.

d) Sistemas de incentivos y técnicas de gestión

En la mayoría de las empresas de la región los trabajadores son remunerados todavía sobre la base de salarios fijos que dependen del tiempo de permanencia en el lugar de trabajo y que a menudo están vinculados a la evolución del salario mínimo. En algunas empresas hay también sistema de pago a destajo.

Por otro lado, la meta de mejorar la productividad y la calidad ha conducido a muchas empresas a explorar nuevos sistemas de remuneración. Un número creciente de empresas entrevistadas en el curso de la investigación están probando nuevas formas de pago a los trabajadores de la producción con miras a elevar su productividad. Por ejemplo, la mayoría de las empresas exportadoras estaban utilizando nuevos sistemas de remuneración a sus trabajadores de producción, que eran innovadores al menos en relación con las prácticas habituales en la región: entre ellos figuran los incentivos por asistencia y logro de calidad, así como las metas de productividad basadas en estándares internacionales.

Estas iniciativas se daban con mayor frecuencia en México. La mitad de las empresas entrevistadas en ese país aplicaban sistemas de pago innovadores en los que los incentivos por asistencia y calidad podían llegar hasta la mitad del salario mensual de los trabajadores. El propósito era claramente encontrar el sistema que más ayudara a las empresas a mejorar tanto su productividad como la calidad del producto. Hay incluso unas pocas empresas mexicanas que pagan un bono extraordinario en función de la capacidad y buena disposición del trabajador para trabajar en diferentes puestos dentro de la planta. Asimismo, en visitas de seguimiento a empresas venezolanas a principios de 1995, se vio que las empresas más modernizantes de ese país estaban también capacitando trabajadores para desempeñar tareas múltiples.

Las empresas que estaban introduciendo nuevas formas salariales se hallaban también entre las más dinámicas e innovadoras en otras áreas, como la intro- 
ducción de nuevas técnicas de gestión. De hecho, esta última característica es casi un requisito previo para el pago de incentivos, ya que para establecer sistemas de remuneración más sofisticados que los habituales en América Latina hay que aplicar procedimientos que permitan un control riguroso de la productividad y la calidad del trabajo de cada trabajador. ${ }^{3}$ Los gerentes de estas empresas modernizantes dicen que una de sus ventajas competitivas más importantes es precisamente su administración de recursos humanos. Aunque todavía no está claro cuáles son los sistemas más eficientes, las empresas tienen plena conciencia de que para mejorar la productividad y la calidad se precisan innovaciones en los sistemas de pago de incentivos.

\section{e) Capacitación}

La mayoría de las empresas en Chile y Venezuela impartían muy poca capacitación a sus trabajadores. Esto resultaba sorprendente respecto de Chile, porque las empresas chilenas pueden obtener crédito tributario para cubrir el costo de algunas actividades de capacitación, y aún así los empresarios de ese país manifestaron que las deficiencias del capital humano estaban entre las mayores desventajas competitivas que enfrentaban (Macario, 1995).

Las empresas mexicanas, en cambio, parecían estar mucho más dispuestas a gastar recursos en capacitar a su personal, aunque, en promedio, el monto gastado era bastante reducido. Algunas de las empresas entrevistadas tenían hasta pequeñas escuelas en la planta, que daban no sólo capacitación especializada, sino programas de educación general.

La mayor disposición de las empresas mexicanas a capacitar a sus trabajadores se debe en parte a una combinación de un más bajo nivel educacional de la fuerza de trabajo y de los programas de capacitación que ofrecen los gobiernos (federal y regionales).

Sin embargo, la principal explicación de los mayores esfuerzos de capacitación de las empresas mexicanas está en el deseo de sus gerentes de lograr un aumento sustantivo de productividad para enfrentar las presiones competitivas. Las empresas que invierten más recursos y energía en capacitación son precisamente aquellas que están transformando completamente sus prácticas empresariales.

\footnotetext{
3 Una de las innovaciones observadas con mayor frecuencia es la introducción de sistemas computarizados que permiten el control del flujo de producción y de los inventarios.
}

\section{f) Subcontratación}

Cuando predominaban las políticas de sustitución de importaciones, había empresas en la región que subcontrataban una parte de su producción, pero esto tendía a ser excepcional. La mayoría de las empresas grandes no recibían muchas presiones para disminuir sus costos y tenían una participación de mercado relativamente estable. Si era preciso llevar a cabo nuevas operaciones, éstas por lo general terminaban siendo absorbidas internamente en la empresa. La subcontratación se efectuaba generalmente en tareas muy especializadas o por aumentos inesperados de la demanda.

Esta situación hoy parece haber cambiado radicalmente. Uno de los cambios más importantes observados durante las entrevistas fue el notable incremento de la subcontratación por las empresas. Este aumento es consecuencia de cambios que están ocurriendo tanto a nivel global como en la región. En primer lugar, ha habido un auge considerable de la subcontratación internacional, al establecer las empresas redes dinámicas de carácter mundial gracias a la disminución de los costos de transporte y al avance de las telecomunicaciones (Dicken, 1992). Por su parte, en América Latina, la combinación de los procesos de liberalización del comercio con la apreciación del tipo de cambio ha expuesto a las empresas a presiones competitivas muy fuertes, y muchas de ellas han debido achicarse y disminuir costos. La subcontratación tiene un papel decisivo en las estrategias de sobrevivencia de las empresas, porque les permite bajar costos fijos y reaccionar más rápidamente a cambios en la demanda.

La mayoría de las empresas entrevistadas habían acentuado la subcontratación de operaciones de fabricación en los últimos años. Más aún, esperaban que la subcontratación aumentara significativamente en los próximos años. Algunas subcontrataban la fabricación de productos que antes producían internamente, pero cuyas líneas de producción se habían cerrado. En otros casos, las empresas decidieron centralizar el diseño de los productos, subcontratando la mayor parte del proceso de fabricación.

Si bien al comienzo de la investigación podría haberse previsto un aumento de la subcontratación, sorprende la amplitud de ese aumento en Chile, México y Venezuela. No sólo prevaleció la subcontratación dentro de los países, sino también la subcontratación en el exterior. En algunos casos, la empresa principal subcontrataba a otras de un país vecino con trabajadores más baratos y mejor adiestrados (por ejemplo, empresas venezolanas que subcontrataban operaciones 
en Colombia). En otros casos, las empresas optaban por ganar una ventaja competitiva yendo a la región productora de los bienes que le restaban participación de mercado, es decir, subcontratando directamente en Asia.

Esta práctica, que está siendo adoptada en diversos sectores, está particularmente difundida en la fabricación de vestuario y calzado. Más que aceptar ser desplazadas de América Latina por productos asiáticos, las empresas han decidido subcontratar directamente en Asia al menos algunas líneas de producción.

Mientras algunas empresas han extremado la práctica, cerrando casi la producción local y concentrándose en la distribución, otras - la mayoría - han reaccionado en forma que les permitirá conseguir una mayor flexibilidad a largo plazo: subcontratan producción en las líneas en que son menos competitivas, y tratan de ser muy competitivas en las líneas que mantienen. Así, si hay modificaciones, como depreciación del tipo de cambio, todavía contarán con capacidad de fabricación para incrementar la producción interna. Entre tanto, esta estrategia les permite defender su participación en el mercado interno, especialmente frente al alza del tipo de cambio y al notorio auge de las exportaciones asiáticas, de bajos precios y calidad cada vez mejor.

Esta práctica está más difundida que lo que se da a conocer. A los empresarios no les gusta mencionarla, en México y en Venezuela más que en Chile, porque temen reacciones negativas. Por otro lado, las asociaciones empresariales están exigiendo protección gubernamental contra lo que reclaman como una competencia desleal de los productos asiáticos. Los empresarios tampoco quieren revelar lo que es uno de los elementos claves de su estrategia. Pero de hecho la mayoría de las empresas competitivas de la región están siguiendo esta estrategia, la que es muy razonable, ya que les permite sobrevivir y concentrarse en la manufactura de productos en los que tienen más capacidad de competir.

\section{g) Distribución y venta al detalle}

Para la mayoría de las industrias manufactureras, la distribución y la venta al detalle no habían tenido un papel clave durante el período en que estuvieron vigentes las políticas de sustitución de importaciones destinadas a crear un sector industrial. Se favorecieron las actividades productivas sobre las de distribución y era mucho más fácil obtener crédito subvencionado para plantas industriales que para establecimientos comerciales. Más aún, los industriales esgrimían un gran poder sobre los comerciantes detallistas, los que no tenían muchas opciones para comprar su mercancía. Por ello se dio importancia a la producción más que a la venta al detalle. Aun cuando los fabricantes distribuyeran sus propios bienes a los consumidores, la esencia de la acividad manufacturera estaba en la planta.

Las circunstancias han cambiado radicalmente para las industrias tradicionales de bienes de consumo. Con la apertura de sus economías, los países latinoamericanos han permitido a sus consumidores que elijan entre una gran variedad de productos. La penetración de las importaciones es muy fuerte. Los comerciantes minoristas ya no están limitados a los artículos producidos localmente, lo que ha provocado un cambio en las actividades de los fabricantes.

El cambio se hizo evidente en el curso de la investigación en Chile, México y Venezuela. La mayor parte de los ejecutivos de las empresas modernizantes manifestaron que su preocupación principal era llegar a ser competitivos en la distribución y lograr reconocimiento del mercado para su marca. Dijeron que éste era el factor más importante para la sobrevivencia de las actividades manufactureras, ya que el mercado estaba inundado de bienes de otros países.

Debido a esta meta, junto con disminuir el grado de integración vertical hacia atrás, ha habido un aumento importante de la inversión para incrementar la integración hacia adelante: para muchas empresas manufactureras, asegurar la presencia de su marca en el mercado interno y contar con una sólida estructura de ventas al detalle les permiten multiplicar la probabilidad de sobrevivencia.

Aunque esta situación se advirtió en los tres países, fue más evidente en Chile. La mayoría de los ejecutivos entrevistados en ese país dijeron que asegurar un sistema de ventas al detalle era para ellos la primera prioridad. A veces, la propia empresa es propietaria de las tiendas, otras veces no lo es, pero en ambos casos la venta al detalle es un aspecto crucial de la estrategia empresarial.

Este fenómeno se observó también en México y en Venezuela, pero no fue tan notorio como en Chile. La relativa pequeñez del mercado interno chileno hace que la participación de mercado sea un factor más importante. Por lo demás, Chile liberalizó el comercio exterior antes que México y Venezuela, y muchas empresas chilenas fueron precursoras en reconocer la importancia de la venta al detalle en la estrategia de las empresas manufactureras.

Cualquiera sea la razón principal, el hecho es que mientras la tendencia fue mucho más acentuada en 
Chile, la importancia de establecer redes de distribución sólidas fue también elemento clave de las estrategias de las empresas más competitivas entrevistadas en México y Venezuela. Un ejecutivo venezolano, que administra una empresa muy interesante e innovadora, dijo lo siguiente: "Si yo tuviera recursos disponibles para invertir, abriría tiendas". De la misma forma, el gerente general de una de las empresas mexicanas visitadas, con plantas de fabricación mejor organizadas, cree que su mayor desventaja competitiva es su falta de integración hacia adelante.

Otro indicador de la importancia de esta tendencia es el hecho de que muchas empresas que estaban invirtiendo dedicaban la mayor parte de los recursos a mejorar la distribución de los productos de la empresa, más que a la propia fabricación. De hecho, las innovaciones tecnológicas, como ventas e inventarios en línea, fueron introducidas con la misma finalidad. De igual manera, las empresas tienden frecuentemente a concentrar sus esfuerzos de capacitación en el área de comercialización, más que en los trabajadores de producción.

Más aún, una cadena de distribución sólida permite a los fabricantes distribuir bienes importados, defendiendo así la participación de mercado de su propia marca. Esto les da la oportunidad de ejercer algún control sobre los precios a los que se venden las importaciones competitivas, así como obtener ganancias de sus ventas. Esta estrategia parece ser muy adecuada desde el punto de vista de los fabricantes, ya que permite a las empresas beneficiarse con el auge de las importaciones y seguir manufacturando las líneas de producción que todavía son competitivas, reteniendo simultáneamente su participación de mercado.

\section{h) Empresas exportadoras}

¿Qué diferencias se observaron entre las empresas modernizantes en general y aquellas que exportaban en forma habitual una parte significativa de su producción (más de 15 o 20\%)? En lo que respecta a las ventas y al empleo, las empresas exportadoras incluidas en la investigación eran bastante similares a las que exportaban sólo ocasionalmente, las que exportaban habitualmente un pequeño porcentaje de su producción y las que no exportaban nada. Aunque las empresas exportadoras tendían a mostrar niveles de empleo y de ventas algo más elevados que las que no exportaban, las diferencias no eran significativas entre ambos grupos dentro de una misma industria.

Parece haber un umbral mínimo de ventas bajo el cual es difícil encontrar empresas que exporten en forma habitual. Es probable que esto se deba a los costos fijos de exportación, como la compra de equipos para mejorar la calidad, la captación de clientes en el exterior y los trámites de exportación. Sin embargo, a partir de un determinado nivel, no parece haber una correlación sistemática entre el tamaño de la empresa y la actividad de exportación: aunque al interior de cada industria la mayor parte de las exportaciones las realizan eficientemente empresas grandes, hay empresas medianas que exportan y empresas grandes que no lo hacen. El creciente número de empresas latinoamericanas de tamaño medio que exportan es un fenómeno interesante que merece mayor estudio.

Sin embargo, la diferencia significativa que se observó entre las empresas exportadoras y las no exportadoras era la mayor preocupación de las primeras por el mejoramiento de la calidad. Las empresas exportadoras estaban aumentando las exportaciones de manufacturas conforme a especificaciones de los mercados de exportación, en lugar de simplemente exportar productos que se comercializaban en el mercado interno. La importancia que las empresas exportadoras daban a la calidad se reflejaba en las especificaciones para el diseño de las plantas, así como en lo relativo a capacitación y sistemas de pago de incentivos. Las empresas exportadoras capacitaban con mayor frecuencia que las no exportadoras y habían introducido más innovaciones en los sistemas de remuneraciones.

Exportar y vender en el mercado interno no deben ser vistos como actividades necesariamente contradictorias. De hecho, frecuentemente pueden ser opciones interrelacionadas, especialmente en economías pequeñas como la chilena. Es posible que una empresa necesite exportar para alcanzar una escala de producción que le permita ser competitiva en el mercado interno. La exportación proporciona a las empresas oportunidades de aprender a satisfacer requerimientos de los mercados más exigentes. Este aprendizaje se difunde en el mercado interno. Simultáneamente, una posición sólida en el mercado interno permite a la empresa soportar el costo de incursionar en nuevas actividades.

La investigación lleva a concluir que en las industrias tradicionales de bienes de consumo no hay diferencias significativas entre las empresas modernizantes grandes y medianas que se concentran principalmente en el mercado local y aquellas que exportan. Las únicas excepciones son la importancia de la calidad para las exportadoras y las oportunidades de aprendizaje que esta actividad proporciona. Puede decirse que, en general, las empresas exportadoras son modernizantes, pero no todas las empresas modernizantes exportan. 


\section{4. $\quad$ Flexibilidad}

En las subsecciones anteriores vimos que las empresas manufactureras de bienes de consumo más innovadoras de Chile, México y Venezuela están sufriendo cambios importantes en la forma en que organizan su producción, en la integración vertical y en el abastecimiento de insumos, así como en el estilo de administración y los sistemas de remuneraciones, entre otros aspectos. Las empresas se están adaptando y están aprendiendo a ser competitivas en el nuevo entorno económico que prevalece en América Latina.

Sin embargo, más que una redefinición masiva, de una vez por todas, lo que caracteriza a estas empresas innovadoras es su flexibilidad para mejorar de manera continuada su rendimiento, por ejemplo, gracias a su habilidad para detectar los cuellos de botella más graves. Ahora las transformaciones se llevan a cabo permanentemente, a veces mediante una serie de cambios pequeños. Ellas desembocan, a largo plazo, en una redefinición masiva de las prácticas de producción, pero de naturaleza distinta a la que resulta del esfuerzo efectuado de una sola vez.

No debe subestimarse la importancia de tal flexibilidad, tanto en la producción como en la distribución. Las empresas con los mejores indicadores de éxito (y las tasas más altas de sobrevivencia a choques adversos, como algunas empresas de Venezuela) son las que pueden reaccionar rápidamente y adaptarse a nuevas circunstancias.

Por ejemplo, una de las áreas donde se demuestra esa importancia es en la generación de sistemas de producción más adaptables que permitan a las empresas reaccionar frente a fluctuaciones en la demanda de los consumidores y producir una gran cantidad de bienes con un número reducido de líneas de producción. Los cambios en la producción están cada vez más dirigidos por la demanda, y el tiempo que demoran las empresas en hacer las modificaciones del caso ha disminuido.

La flexibilidad también es crítica en la capacidad de las empresas para reaccionar frente a variaciones del tipo de cambio, reteniendo un mínimo de capacidad productiva para mantener su participación de mercado, y a la vez mostrando disposición a transformarse rápidamente en distribuidores de líneas de productos importados cuando el tipo de cambio se aprecia significativamente. La estrategia de convertirse en importadores principales de productos competitivos con los propios y distribuirlos a través de sus mismos canales de distribución fue adoptada en forma experimental por algunas empresas en Chile a fines de los años setenta y a principios de los ochenta. Retrospectivamente, se aprecia que esta estrategia fue muy exitosa.

Los empresarios han aprendido de su experiencia, y las empresas más exitosas y flexibles de los tres países son las que han podido convertirse rápidamente en importadoras y distribuidoras de productos importados competitivos. Se dieron cuenta de que era la mejor manera de manejar los precios y de fijar los precios de los bienes importados frente a los de fabricación local según sus capacidades de producción. Esta estrategia permite que la empresa, sin interrumpir la producción de sus bienes más competitivos, aproveche sus propios canales para distribuir bienes importados. Tras ella está la idea de que si la empresa no lo hace, otra aprovechará la oportunidad y la desplazará del mercado.

Pese a que transformarse en distribuidores de bienes importados es una estrategia que están adoptando crecientemente los empresarios manufactureros de México y Venezuela, y que sigue practicándose entre los chilenos, ellos no siempre lo reconocen, ya que se resisten a admitir que están transformándose en grandes importadores de bienes competidores.

\section{El cambio en la esencia de la actividad manu- facturera}

Otro rasgo importante de los cambios que están ocurriendo en el comportamiento de las empresas es la modificación de la esencia de su estrategia.

En el régimen de sustitución de importaciones las empresas manufactureras tenían una posición relativamente ventajosa con respecto a las empresas distribuidoras y al comercio al detalle: entre otras cosas, podían obtener créditos a tasas preferenciales y permisos especiales para importar insumos y podían cabildear para detener la importación de bienes competidores una vez establecidas sus plantas locales. Aunque algunas empresas manufactureras fueran a la vez detallistas, la esencia de la actividad manufacturera estaba en la producción.

La situación de las empresas manufactureras latinoamericanas es hoy muy distinta. Enfrentan una competencia vigorosa en el mercado interno, tanto de importaciones como de otras empresas locales que tratan de defender su participación de mercado. Los consumidores son ahora más exigentes por haber tenido acceso a una mayor variedad de bienes, no sólo como resultado de la liberalización del comercio, sino también por la apreciación del tipo de cambio. 
CUADRO 1

\section{Resumen de las estrategias de las empresas manufactureras en el decenio de 1990}

\begin{tabular}{|c|c|}
\hline & Empresas modernizantes \\
\hline \multirow[t]{9}{*}{$\begin{array}{l}\text { Areas en las que las dife- } \\
\text { rencias son muy significa- } \\
\text { tivas }\end{array}$} & $\begin{array}{l}\text { Flexibilidad: introducción permanente de cambios en la producción y } \\
\text { la distribución frente a cambios en la demanda y en el entorno } \\
\text { macroeconómico (como en el tipo de cambio) }\end{array}$ \\
\hline & Contratación de consultores externos para modernizarse \\
\hline & Se mide la productividad \\
\hline & Sistema de pago de incentivos \\
\hline & Gestión profesional \\
\hline & Cambios en la producción orientados por la demanda \\
\hline & $\begin{array}{l}\text { Grandes aumentos en la subcontratación basados en relaciones de lar- } \\
\text { go plazo }\end{array}$ \\
\hline & Fuerte interacción entre producción y distribución \\
\hline & $\begin{array}{l}\text { Cuando es posible, vínculos fuertes con detallistas o aumentos signi- } \\
\text { ficativos de las ventas propias al detalle }\end{array}$ \\
\hline $\begin{array}{l}\text { Areas en las que las dife- } \\
\text { rencias son significativas }\end{array}$ & $\begin{array}{l}\text { Importante disminución de la integración vertical: las plantas relacio- } \\
\text { nadas se transforman en empresas independientes }\end{array}$ \\
\hline
\end{tabular}

Empresas no modernizantes

Comportamiento rígido y pasivo

Dependencia exclusiva del expertizaje interno

No hay datos de productividad

Sistemas de remuneración tradicionales

Gestión de estilo familiar

Cambios limitados en respuesta a problemas de producción

La subcontratación no existe o es muy limitada

La empresa se centra exclusivamente en la producción

Vínculos débiles con detallistas

Disminución de la integración vertical

Aumento significativo de la utilización de insumos importados

Aumento de utilización de insumos importados

Esfuerzos por mejorar la capacidad de diseño

Capacidad de diseño limitada

Disminución de inventarios

Grandes volúmenes de inventarios

Cambios habituales en el diseño de los procesos productivos

Diseño de procesos rígidos

Reducción del número de líneas de producción

Se mantiene el número de líneas de producción

Mayor diversidad de productos en las líneas de producción

Diversidad limitada de productos en las líneas de producción.

La calidad importa

La calidad no es prioritaria

Responsabilidad de cada trabajador por la calidad

Control de calidad en pocos puntos de la línea de producción

Se introducen innovaciones tecnológicas en puntos claves, luego de evaluar cuellos de botella

Si se introducen innovaciones tecnológicas, se hace en paquetes, no como resultado de una evaluación cuidadosa de las fortalezas y debilidades de la planta

Se introducen innovaciones tecnológicas en forma habitual y permanente

Las innovaciones tecnológicas se introducen "de una vez por todas"

La empresa capacita a sus trabajadores

La empresa no capacita a sus trabajadores 
Hoy, una empresa manufacturera competitiva no sólo tiene que modernizar la organización de la producción, sino que también debe desarrollar una estrategia activa y agresiva en la venta al detalle. La liberalización del comercio, particularmente en un contexto de apreciación cambiaria, ha acrecentado significativamente la importancia de la distribución y la venta al detalle. Las empresas manufactureras más innovadoras de la incluidas en la investigación, eran las que tenían redes de tiendas minoristas para preservar la participación en el mercado interno y beneficiarse con la venta de bienes importados. La venta al detalle permite también a las empresas manufactureras aumentar sus márgenes de utilidad, ya que es en esencia una actividad no transable cuyos márgenes de utilidad se han elevado en los últimos años. También les permite manejar de manera flexible una mezcla de bienes manufacturados en sus propias plantas y de bienes importados, dependiendo de la evolución del tipo de cambio.

Las entrevistas de seguimiento hechas en empresas venezolanas en 1995 mostraron que las empresas más competitivas estaban poniendo en práctica la estrategia de disminuir la cantidad de bienes que vendían a intermediarios y aumentar la proporción de bienes que ellas mismas vendían al detalle. Los ejecutivos de esas empresas opinaban que la clave para la sobrevivencia de sus empresas radicaba en mejorar la red de distribución y vender ellas mismas al detalle la mayoría de los productos.

La transformación de la forma en que las economías latinoamericanas se integran a los mercados mundiales ha tenido importantes efectos en el comportamiento de las empresas manufactureras. Ellas necesitan actualizar sus capacidades de oferta en respuesta a los cambios de la demanda, pero para llevar a cabo esa actualización, que es decisiva para su sobrevivencia, deben ser capaces primero de defender su presencia en el mercado. Lo que ha cambiado con la liberalización del comercio, la globalización y las transformaciones del entorno económico de las empresas es precisamente la esencia de la acividad manufacturera: para sobrevivir y ser competitivas, las empresas manufactureras deben mejorar su capacidad de comercialización y, para ello, deben transformar sus capacidades de oferta.

\section{Incertidumbre}

La estabilidad macroeconómica y la certeza de que la política económica se mantendría estaban entre las características positivas más importantes del entorno económico que mencionaron los empresarios chilenos y mexicanos en las entrevistas que se realizaron en 1993, antes de la crisis mexicana que empezó con la devaluación de diciembre de 1994.

En contraste, para las empresas venezolanas entrevistadas en 1993 la incertidumbre que rodeaba a las políticas económicas era uno de los principales obstáculos para invertir en proyectos de modernización y de exportación. Esto se confirmó en las entrevistas de seguimiento de 1995: las empresas que habían adoptado políticas activas de modernización estaban en una posición extremadamente vulnerable o en bancarrota, mientras que las que habían adoptado una actitud pasiva se habían beneficiado con la decisión de no modernizarse.

Los casos específicos de los dos más grandes fabricantes de vestuario entrevistados en Venezuela, en adelante las empresas A y B, son ilustrativos al respecto. Cuando se hicieron las primeras entrevistas (marzo de 1993), estas dos empresas habían decidido adoptar dos estrategias opuestas: la empresa A había decidido que seguiría operando básicamente de la misma forma como lo había hecho bajo el régimen de sustitución de importaciones y que no invertiría en mejorar la capacidad de producción ni la calidad de los bienes. Esta estrategia conservadora se adoptó también para las exportaciones, en las que la empresa decidió no invertir recursos.

La empresa B, en cambio, había decidido invertir una cantidad considerable de recursos en el mejoramiento integral de la capacidad de producción de la empresa, tanto en términos de calidad de los bienes producidos como del volumen de producción. Para lograrlo, contrató a consultores internacionales que rediseñaron la planta y capacitaron a los trabajadores. Simultáneamente se introdujeron innovaciones técnicas con la compra de nuevos equipos y se transformaron los procedimientos de producción. El objetivo de la empresa era hacer frente a la fuerte competencia de las importaciones en el mercado local y luego seguir expandiendo sus mercados con exportaciones.

Al momento de las entrevistas iniciales, parecía que la empresa A moriría lentamente a causa de la creciente competencia de las importaciones y de la incapacidad (o falta de interés de sus propietarios) para reaccionar al cambio del modelo de sustitución de importaciones por uno de economía abierta. En contraste, parecía que la estrategia de la empresa B le permitiría desarrollar su competitividad en el nuevo entorno, sobrevivir a la competencia de las importa- 
ciones en el mercado interno y exportar en forma regular.

Dos años después, en las entrevistas de seguimiento de 1995, se comprobó que las circunstancias eran sorprendentemente distintas. El nuevo gobierno de Venezuela había decidido revertir diversas reformas de política emprendidas a fines del decenio de 1980 y comienzos del siguiente. Aunque la política de liberalización del comercio no fue descartada formalmente, lo fue de hecho al aplicarse controles cambiarios que dificultaron a las empresas el acceso a divisas para importar, incluso insumos. La competencia de las importaciones había sido así anulada. Más aún, la fijación del tipo de cambio en momentos de alta inflación hizo muy difícil que las empresas manufactureras exportaran.

En esas circunstancias, la empresa A tuvo un éxito relativo gracias a su decisión de no invertir en la modernización de su planta. Esta estrategia le había permitido tener una posición financiera sólida sin incurrir en deudas. Fue exitosa porque los cambios de política económica, así como la introducción de controles cambiarios, habían reducido de manera importante la competencia de las importaciones, mientras simultáneamente subía la tasa de interés. De esta forma, las empresas que habían tenido una actitud pasiva y habían decidido no cambiar su estrategia y no modernizarse, se beneficiaron de la disminución de la competencia de las importaciones y de una buena posición financiera.

En cambio, las empresas que habían invertido en mejorar su capacidad de producción se vieron afectadas por el alza de las tasas de interés. La empresa B estaba en graves problemas financieros, pues había incurrido en deudas para financiar su modernización. Aunque todavía era capaz de exportar, esta actividad se había visto afectada por obstáculos para importar insumos competitivos de buena calidad, como los controles cambiarios y la apreciación del tipo de cambio. La existencia de procedimientos administrativos complejos y discrecionales contribuyó también a hacer muy difícil importar y exportar. De ahí que las empresas que habían invertido en modernizarse y que habían tratado de ser competitivas perdieran finalmente en comparación con las que habían adoptado una actitud pasiva.

Está claro que esta situación no perdurará. Venezuela no puede soportar indefinidamente un tipo de cambio artificial ni podrá mantener siempre políticas que limiten la competencia de las importaciones. Las empresas venezolanas se encontrarán algún día nuevamente en un entorno competitivo. En estas circunstan- cias, la empresa A se verá claramente obligada a cambiar para no perder una importante participación de mercado, y la empresa B cosechará el beneficio de sus inversiones.

Mientras tanto, no cabe duda de que la empresa B ganará participación de mercado aun si la economía no se abre, gracias a sus esfuerzos de modernización. Cuando fue entrevistada en 1995, esta empresa estaba también aumentando su participación de mercado como fruto de la quiebra de muchas empresas locales. Lo que cabe preguntarse es si las empresas que invirtieron en modernizarse estarán funcionando en el largo plazo o si deberán cerrar debido a la carga financiera de la inversión y a la reversión de la política económica.

Más aún, las empresas cuyos ejecutivos creyeron en las aseveraciones del gobierno de que la liberalización del comercio se mantendría y que debían invertir en proyectos de exportación, han sido las más afectadas con esta vuelta atrás de la política económica. La revaluación de la moneda derivada de los controles cambiarios y la fijación de tipos de cambio en medio de un proceso inflacionario hicieron que sus exportaciones resultaran menos competitivas en los mercados externos. Los obstáculos para la importación de insumos que suponían los controles cambiarios y la tramitación aduanera, eran dificultades adicionales para las empresas que, como parte de sus estrategias de modernización, habían aumentado sus importaciones de esos bienes.

Las entrevistas realizadas durante la investigación muestran que la incertidumbre en torno a la política económica se traduce en una reducción considerable de la inversión de las empresas. No hay incentivos para que inviertan en proyectos de largo plazo cuando la inestabilidad macroeconómica y la posibilidad de echar pie atrás en la política hacen impredecible la rentabilidad.

La comparación del comportamiento de las empresas en los tres países incluidos en la investigación muestra que la incertidumbre es la razón más importante por la que muchas empresas se resisten al cambio, o no cambian más rápidamente. La incertidumbre tiene un doble efecto negativo en las decisiones de los empresarios para transformar sus empresas: hay incertidumbre sobre lo que deben hacer, y hay incertidumbre respecto de la sustentabilidad de la política económica. Los empresarios a menudo están conscientes de que deben cambiar la forma de funcionamiento de sus empresas, pero no están seguros de qué cambios impulsar y cómo hacerlo. Esto muestra la importancia de 
proporcionar a las empresas apoyo técnico en prácticas de administración a través de redes públicas o privadas. Por otra parte, la incertidumbre que probablemente afecta más negativamente las decisiones de modernización de las empresas es la de no saber si la política de liberalización se mantendrá o no. Si esta política no tiene plena credibilidad, muchas empresas serán renuentes a cambiar su comportamiento. La indecisión puede significar que cierren muchas más empresas que lo que se podría esperar.

\section{IV}

\section{Conclusiones}

Las empresas manufactureras de América Latina están experimentando transformaciones importantes para llegar a ser competitivas en las nuevas condiciones del mercado, que se caracterizan por la liberalización comercial, la globalización y, en general, por la transformación del entorno económico. Muchas empresas todavía tienen una actitud pasiva o reactiva, con un comportamiento similar al que se describe en la sección II. Sin embargo, la abrumadora mayoría de los empresarios de la región están conscientes de que deben cambiar su forma de funcionamiento y ya han comenzado a hacerlo: el comportamiento de la mayoría de las empresas es muy distinto hoy de lo que era hace 10 ó 15 años.

En estos cambios de comportamiento de las empresas ha influido con fuerza la transformación de la política macroeconómica y comercial, así como la del entorno económico general. Muestra de ello son las diferencias de comportamiento de las empresas entre los países.

No obstante, la investigación mostró que había empresas en los tres países que estaban cambiando sus comportamientos al margen del entorno económico, con la adopción de estrategias muy agresivas e innovadoras. Mientras que, en promedio, las empresas venezolanas tendían a ser más innovadoras y reacias al cambio que sus contrapartes chilenas y mexicanas, aquellas más competitivas tenían estrategias muy similares a las más innovadoras de Chile y México. Hay mucho que aprender del comportamiento de estas empresas, especialmente para formular recomendaciones de política.

En general, no se observaron diferencias entre las empresas exportadoras y las modernizantes que esta-
Por lo tanto, para que las empresas cambien su comportamiento y procuren ser más competitivas, es de la mayor importancia que puedan confiar en los grandes cambios de política. Por otro lado, también es posible que, ante la incertidumbre, sean más las empresas que no cambien, o que lo hagan con más lentitud. Para modernizar se necesita la voluntad de los gerentes de modificar las prácticas de sus empresas y de invertir fuertemente, así como la certeza de que las nuevas políticas serán permanentes. ban orientadas al mercado interno, salvo que las primeras parecían preocuparse más de mejorar la calidad y tenían mayores posibilidades de aprendizaje que las no exportadoras. Sin embargo, la investigación mostró que la modernización de la empresa es condición previa para mantener una actividad exportadora regular.

La transformación que ha tenido lugar en lo que se considera la esencia de la actividad manufacturera es una de las conclusiones más importantes de la investigación. Por lo menos para las empresas manufactureras de bienes de consumo más innovadoras, la esencia de esta actividad ya no se halla en los aspectos de producción, sino en un punto en que se combina la manufactura con la distribución de los bienes producidos, y frecuentemente con la de otros bienes importados y nacionales también. Este es el punto del conjunto producción-distribución en que las empresas manufactureras tienen mayores posibilidades de acrecentar sus utilidades y a la vez defender su participación de mercado. La estrategia que permita que las empresas tengan éxito en este aspecto crucial será determinante para asegurar la competitividad de las empresas manufactureras de América Latina en los próximos años.

Los cambios que están ocurriendo en las economías de la región han tenido consecuencias importantes en las empresas manufactureras y han traído consigo modificaciones significativas en su comportamiento. Entender mejor estos cambios es un requisito previo para comprender las tendencias, así como para formular recomendaciones de política que busquen mejorar la competitividad de las empresas.

Las empresas innovadoras han adoptado un comportamiento flexible y están mejorando su capacidad 
de producción y de comercialización. Han introducido importantes cambios en materia de integración vertical, abastecimiento de insumos, innovaciones tecnológicas, sistemas de incentivos, técnicas de administración, capacitación y subcontratación, así como de distribución y venta al detalle.

Sin embargo, hasta los empresarios modernizantes sintieron con frecuencia que necesitaban intensificar el esfuerzo por mejorar sus empresas. El costo de hacerlo y, más importante aún, las dificultades para obtener información apropiada sobre las mejores prácticas de una determinada industria, han sido obstáculos para la introducción acelerada de transformaciones en gran escala. Estas restricciones evidentemente fueron más grandes para las empresas no modernizantes. Sus gerentes sabían que tenían que cambiar su forma de operar, pero no sabían bien qué necesitaba hacerse y cómo. Mientras algunos empresarios de la región permanecerán pasivos y mientras les sea posible seguirán funcionando como lo hacían al amparo del régimen de sustitución de importaciones, hay muchos a los que les gustaría modernizar sus empresas pero que carecen de la información necesaria para hacerlo.

Esto sugiere que el gobierno tiene un papel que cumplir en el apoyo a programas que entreguen a las empresas información acerca de las mejores prácticas, sobre todo en diseño de plantas, mejoramiento de la calidad, sistemas de incentivos, capacitación, etc. Una actividad clave, que crea muchas externalidades, es el de proporcionar a las empresas información sobre los mercados de exportación.

Una forma eficaz de complementar información insuficiente es la de crear centros tecnológicos para industrias específicas destinados a mejorar la capacidad de oferta de las empresas. Tales centros deberían cubrir las áreas donde es crucial la innovación, como las descritas anteriormente; los gobiernos deberían auspiciarlos, pero su creación debería hacerse en conjunto con el sector privado, en particular con las asociaciones empresariales. Estos centros también podrían promover la asociación entre empresas para obtener mejores condiciones de aprovisionamiento de insumos, asistencia técnica y distribución que las que podrían obtener por separado. Buen ejemplo de estos centros es el fundado en Colombia por la Asociación Colombiana de Industrias Plásticas (ACOPLASTICOS), o el Centro de Productividad Industrial (CEPRI) que se estableció hace poco en Chile con el apoyo del gobierno.

Por último, hay varios criterios importantes que se deben tener presentes al considerar la formulación de políticas:

i) la función más importante de la política es la de crear un ambiente económico estable en el que las empresas puedan planificar sus inversiones a largo plazo. Como se vio antes, la incertidumbre promueve comportamientos no productivos;

ii) las políticas deberían diseñarse para ser aplicadas con eficacia y estar sujetas a evaluación periódica. Si no se hace así, pueden resultar ineficaces y generar desconfianza hacia el gobierno en el sector privado;

iii) las políticas deberían propender explícitamente a la modernización de las empresas y no ser un pretexto para el retorno al proteccionismo, y

iv) para que sean eficaces, las políticas deben diseñarse y aplicarse en estrecha coordinación con el sector privado.

El nuevo marco económico que rige en los países de América Latina y el aumento de la globalización en el mundo, constituyen un desafío y una oportunidad para la mayoría de las empresas manufactureras de la región. Muchas empresas han transformado su comportamiento por completo. Otras no van a cambiar, cualquiera sea el entorno de política en que se encuentren. Hay un tercer grupo, compuesto por la mayoría de las empresas manufactureras, cuyos gerentes desean cambiar e introducir cambios. Este es el grupo al que debiera apuntar la política económica si lo que se desea es que la región tenga la capacidad de crecer a tasas razonablemente altas.

\section{Bibliografía}

Baumann, R. (1994): Exporting and the Saga for Competitiveness of Brazilian Industry:1992, Working paper, $\mathrm{N}^{\circ} 27$, Santiago de Chile, Comisión Económica para América Latina y el Caribe (CEPAL)

Bielschowsky, R. (1994): Two Studies on Transnational Corporations in the Brazilian Manufacturing Sector: The 1980s and early 1990s, serie Desarrollo productivo, $\mathrm{N}^{\circ} 18$, Santiago de Chile, CEPAL.
Castillo, M., M. Dini y C. Maggi (1994): Reorganización industrial y estrategias competitivas en Chile, LC/R.1467, Santiago de Chile, CEPAL.

CEPAL (1994): Reestructuración y desarrollo productivo: desafío y potencial para los años noventa, Estudios e informes de la CEPAL, No 92, LC/G.1831-P, Santiago de Chile. Publicación de las Naciones Unidas, $\mathrm{N}^{\circ}$ de venta S.94.II.G.4.

Corbo, V. y J. M. Sánchez (1984): Impact on firms of the liberalisation and stabilisation policies in Chile: Some case studies, 
Documento de trabajo, $\mathrm{N}^{\circ}$ 91, Santiago de Chile, Pontificia Universidad Católica de Chile, Instituto de Economía.

Dicken, P. (1992): Global Shift: The Internationalization of Economic Activity, Londres, Paul Chapman Publishing Ltd.

Katz, J. y G. Burachik (1997): La industria farmacéutica y farmoquímica argentina en los años noventa, J. Katz (comp.), Apertura económica y desregulación en el mercado de medicamentos, Buenos Aires, CEPAL/Centro Internacional de Investigaciones para el Desarrollo (CIID)/Alianza Editorial.

Macario, C. (1995): Competitividad de las empresas latinoamericanas: comportamiento empresarial y políticas de promoción de exportaciones, Documento de trabajo, $\mathrm{N}^{\circ} 38$, Santiago de Chile, CEPAL. (1998a ): Restructuring in Manufacturing: Case Studies in Chile, Mexico and Venezuela, serie Desarrollo productivo, $\mathrm{N}^{\circ} 44$, Santiago de Chile, CEPAL.

(1998b): Why and How do Manufacturing Firms Export: Evidence from Successfull Exporting Firms in Chile, Colombia and Mexico, Tesis doctoral, Columbia, Missouri, University of Missouri.

Nelson, R.R. (1991): Why do firms differ, and how does it matter, Strategic Management Journal, vol. 12, Nueva York, John Wiley \& Sons Ltd.

Schmitz, H. (1995): Small shoemakers and fordist giants: Tale of a supercluster, World Development, vol. 23, $\mathrm{N}^{\circ} 1$, Oxford, Reino Unido. 